\title{
Manejo endoscópico de anomalías del cuarto arco branquial: Reporte de tres casos
}

\section{Endoscopic management of anomalies of the fourth branchial arch: Report of three cases}

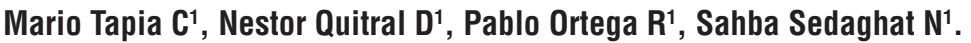

\begin{abstract}
RESUMEN
Las anomalías de cuarto arco branquial corresponden a una entidad patológica infrecuente. Para su manejo existen distintas alternativas terapéuticas siendo una de ellas la cauterización endoscópica.

Reportamos 3 casos de senos de cuarto arco branquial tratados mediante cauterización endoscópica en el Hospital Regional de Concepción luego de una revisión de fichas clínicas de todos los pacientes con diagnóstico de anomalías de cuarto arco branquial. Se identificaron tres casos de senos de cuarto arco branquial. Todos corresponden a pacientes de sexo masculino que presentaron cuadro de absceso cervical, diagnosticándose 2 de ellos al presentar recurrencia. Todos fueron tratados mediante cauterización endoscópica de la apertura fistulosa en seno piriforme. Estas anomalías representan vestigios de un trayecto que se origina desde el vértice del seno piriforme. La cauterización endoscópica presenta una serie de ventajas con tasas de recurrencia similares a la cirugía abierta de cuello, menores tasas de complicaciones y costo económico. Las anomalías de cuarto arco branquial constituyen una patología infrecuente y el diagnóstico requiere alta sospecha clínica. El manejo endoscópico ha demostrado ser una alternativa segura y efectiva con menor tasa de complicaciones.
\end{abstract}

Palabras clave: Seno piriforme, absceso, región branquial, fístula.

\begin{abstract}
Anomalies of the fourth branchial arch correspond to an uncommon pathological entity. There are different therapeutic alternatives being one of them the endoscopic cauterization. We report 3 cases of fourth branchial arch anomalies treated by endoscopic cauterization in the Regional Hospital of Concepción. Review of clinical records of all patients with diagnosis of fourth branchial anomalies operated by endoscopic cauterization at the Regional Hospital of Concepción. Cases: Three cases of fourth branchial arch
\end{abstract}

Médicos del Servicio de Otorrinolaringología y Cirugía de Cabeza y Cuello, Hospital Guillermo Grant Benavente, Concepción, Chile. 
sinus were identified. All of them were male patients who presented with a cervical abscess, diagnosing 2 of them when they recurred. All 3 cases were treated by endoscopic cauterization of the fistulous opening in the piriform sinus. These anomalies represent vestiges of a path that originates from the apex of the piriform sinus. Endoscopic cauterization presents a number of advantages with recurrence rates similar to open neck surgery, with lower complication rates and economic cost. Fourth branchial anomalies constitute an uncommon pathology and the diagnosis requires high clinical suspicion. Endoscopic management has proven to be a safe and effective alternative with a lower rate of complications.

Key words: Pyriform sinus, Abscess, Branchial region, Fistula.

\section{INTRODUCCIÓN}

Las anomalías branquiales se originan por un desarrollo anormal del complejo embriológico conocido como aparato branquial. Los arcos branquiales dan origen a una serie de estructuras y entre las que se desarrollan a partir del cuarto arco se incluyen los cartílagos laríngeos, los músculos constrictores faríngeos y laríngeos, el nervio laríngeo superior, la aorta torácica izquierda, la arteria subclavia derecha proximal y las glándulas paratiroides superiores ${ }^{1}$. La mayoría de los quistes, fístulas y senos cervicales laterales son considerados originados del aparato branquial. Los quistes branquiales no tienen apertura externa 0 visceral, mientras que los senos branquiales se comunican con la piel 0 el lumen faríngeo, y las fístulas branquiales son trayectos que conectan la faringe con la piel. La primera publicación de una lesión de una anomalía branquial fue en 1832 por Ascherson y cols ${ }^{2}$, y posteriormente, Raven y cols $^{3}$ reportaron el primer caso de fístula de seno piriforme.

Las anomalías del cuarto arco branquial presentan distintas manifestaciones clínicas, las más frecuentes son mediante un cuadro de absceso cervical recurrente 0 tiroiditis aguda supurativa ${ }^{4-6}$. La prevalencia reportada para anomalías del cuarto arco branquial es baja, con menos de 100 casos publicados según la mayoría de los reportes ${ }^{7}$, I0 que representa $1 \%$ a $4 \%$ de todas las anomalías branquiales ${ }^{8}$. Por lo tanto, las recomendaciones en cuanto a su diagnóstico y manejo aún no cuentan con un alto nivel de evidencia. El manejo tradicionalmente involucra la resección del trayecto del seno, asociado a tiroidectomía parcial si la glándula tiroidea está involucrada y además canulación de la fístula del seno piriforme si ésta es identificada mediante laringoscopía directa y de esta manera ayude a lograr una resección total ${ }^{9}$. Otra alternativa terapéutica corresponde a la cauterización endoscópica de las anomalías de cuarto arco branquial con fístula en el ápex del seno piriforme que ya ha demostrado ser una técnica efectiva y con baja tasa de complicaciones ${ }^{10}$. En este trabajo, reportamos 3 casos de senos branquiales del cuarto arco tratados mediante laringoscopía directa y cauterización endoscópica en el Hospital Regional de Concepción.

Estudio retrospectivo, descriptivo y transversal. Revisión de fichas clínicas de todos los pacientes con diagnóstico de quiste de cuarto arco branquial operados mediante cauterización endoscópica en el Servicio de Otorrinolaringología y Cirugía de Cabeza y Cuello en el Hospital Regional de Concepción entre enero del año 2013 y enero del año 2017.

\section{Caso 1}

Paciente masculino de 3 años 11 meses de edad, consulta en octubre de 2013 por cuadro de 5 días de otalgia izquierda y compromiso del estado general que evolucionó con aumento de volumen cervical anterior en relación al músculo esternocleidomastoideo (ECM) izquierdo. Destaca al ingreso aumento de parámetros inflamatorios. Se realiza tomografía axial computarizada (TAC) de cuello con contraste, evidenciándose una colección de 5 por 4 centímetros a nivel infrahioideo izquierdo, asociado a desviación de tráquea hacia contralateral.

El paciente es hospitalizado para manejo con antiobioticoterapia endovenosa y drenaje quirúrgi- 
co, drenándose aproximadamente 15 centímetros cúbicos (cc) de pus. El paciente completa 10 días de tratamiento antibiótico endovenoso con evolución favorable y recibe alta hospitalaria. Sin embargo, en julio del año 2014 el paciente presenta cuadro de odinofagia asociado a aumento de volumen cervical doloroso y fluctuante a la palpación, en relación a zona de cicatriz de cirugía previa. Se realiza TAC cervical con contraste, en el cual se evidencia nuevamente una colección de 4 por $5 \mathrm{~cm}$ de diámetro mayor. Se trató nuevamente mediante drenaje quirúrgico, y se realiza adicionalmente una laringoscopía directa que evidencia una apertura fistulosa a nivel de ápex de seno piriforme izquierdo con salida de pus, compatible con fístula de cuarto arco branquial. Se realiza cauterización con aguja de tungsteno Colorado del trayecto fistuloso, sin incidentes. El paciente presentó una evolución favorable, con controles periódicos sin recurrencia hasta la fecha.

\section{Caso 2}

Paciente masculino de 18 años de edad, presenta cuadro de aumento de volumen cervical izquierdo asociado a fiebre y aumento de parámetros inflamatorios durante el mes de enero del año 2016. Al ingreso se realiza TAC de cuello y tórax con contraste, en el cual se visualiza una colección cervical izquierda sugerente de absceso. Se realiza cervicotomía de urgencia de colección multitabicada con abundante secreción purulenta. Evoluciona favorablemente; tras 11 días de antibioticoterapia endovenosa y con TAC de cuello

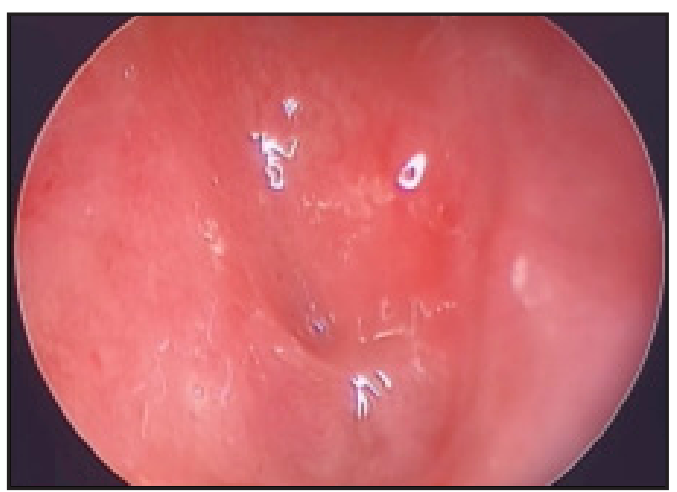

Figura 1. Laringoscopía directa y visión endoscópica de seno piriforme izquierdo. Apertura de quiste de cuarto arco branquial en vértice de seno piriforme. con contraste de control sin evidencia de colección residual, el paciente es dado de alta.

Siete meses después, el paciente cursa con infección de vía respiratoria alta que evoluciona con aumento de volumen cervical en relación a extremo distal de cervicotomía previa. Por el antecedente de absceso cervical se realiza nasolaringofibroscopía, en la cual se observa edema discreto de seno piriforme izquierdo. Por antecedente, localización e imagenología se sospechó quiste de cuarto arco branquial sobreinfectado. Se realiza panendoscopía identificándose fístula en ápex de seno piriforme izquierdo que al comprimir superficialmente la región cervical presenta salida de pus, se repite compresión hasta el cese de salida y finalmente se cauteriza fístula con aguja monopolar. Evoluciona favorablemente sin complicaciones, actualmente en control periódico sin recurrencia hasta la fecha.

\section{Caso 3}

Paciente masculino de 11 años de edad sin antecedentes mórbidos. Derivado desde hospital periférico en agosto de 2016 por cuadro de 12 días de evolución caracterizado por cervicalgia y fiebre intermitente, que evoluciona con aumento de volumen cervical anterior y adenopatías cervicales bilaterales. Destaca al ingreso aumento de parámetros inflamatorios. Evaluado inicialmente por pediatría es hospitalizado por sospecha de tiroiditis aguda. Se estudia mediante ecografía cervical que describe lesión heterogénea e irregular en relación a espacio visceral anterolateral izquierdo, sin plano de clivaje tiroideo y hormonas tiroideas dentro de rangos normales. Se solicitó evaluación por otorrinolaringología complementándose estudio con TAC cervical, el cual evidencia aumento de densidad de tejido adiposo adyacente a lóbulo tiroideo izquierdo, con extensión hasta la porción caudal de seno piriforme asociado a colección de 1,6 por $1 \mathrm{~cm}$, sugerente de absceso. También se estudió mediante una nasolaringofibroscopía en la cual fue posible observar edema del seno piriforme izquierdo. Por sospecha de anomalía de cuarto arco branquial, se realiza panendoscopía identificándose orificio en ápex de seno piriforme izquierdo de aproximadamente 3 milímetros con salida de secreción purulenta (Figuras 1 y 2). Se realiza drenaje por compresión cervical manual 

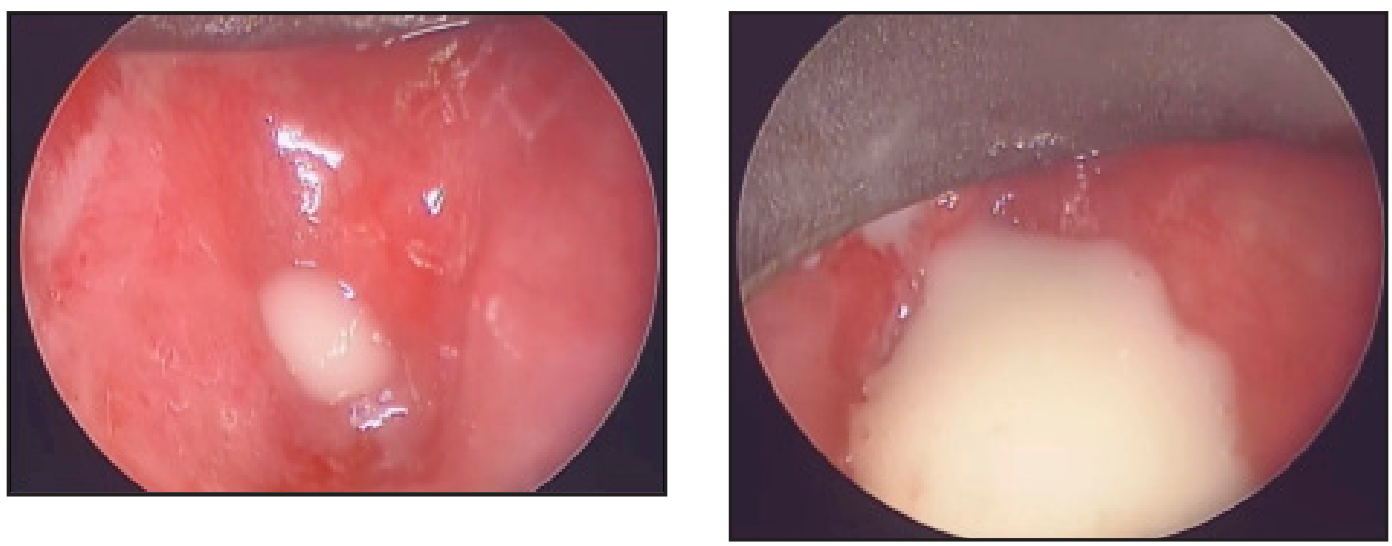

Figuras 2 y 3. Laringoscopía directa y visión endoscópica de seno piriforme izquierdo. Secreción purulenta desde apertura fistulosa en vértice de seno piriforme.

hasta cese de salida de pus y posteriormente se cauteriza con aguja monopolar sin incidentes perioperatorios (Figura 3). El paciente evolucionó favorablemente, actualmente en control periódico con 6 meses de seguimiento sin recurrencia.

\section{DISCUSIÓN}

Las anomalías del cuarto arco branquial representan vestigios de un trayecto que se origina desde el vértice del seno piriforme. Este se desplaza inferiormente por la ranura traqueoesofágica en una posición posterior a la glándula tiroides, y en el tórax realiza un giro inferior respecto a la aorta en el lado izquierdo y a la arteria subclavia en el lado derecho.

Su región descendente antes del primer giro es la localización más frecuente de infección clínica. Posteriormente el trayecto cambia de dirección hacia superior y posterior a la arteria carótida común hasta alcanzar el nervio hipogloso, donde realiza un segundo giro alrededor de éste y termina su curso en el borde medial del músculo esternocleidomastoideo ${ }^{12}$.

Resulta difícil diferenciar entre anomalías del tercer y cuarto arco, sin embargo, estos 2 tipos de anomalías son anatómicamente distintas. Las anomalías del tercer arco branquial se originan desde la base del seno piriforme y pasan sobre el nervio laríngeo superior. En contraste, el trayecto de las anomalías del cuarto arco branquial se originan en

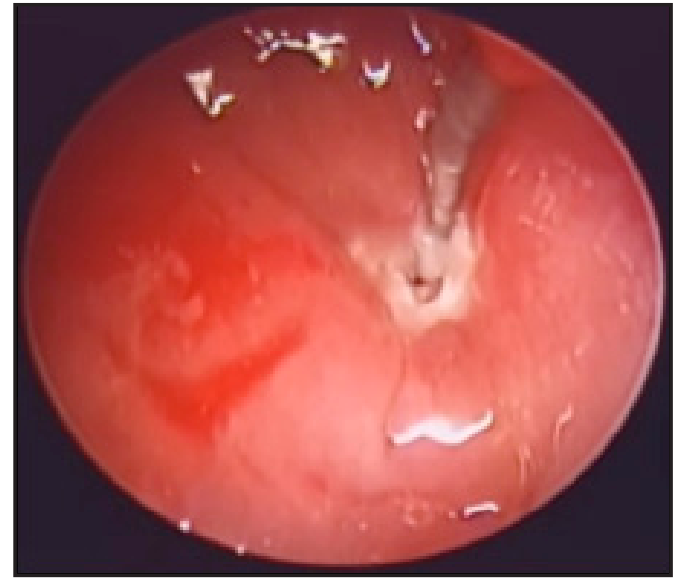

Figura 4. Laringoscopía directa y visión endoscópica de seno piriforme izquierdo. Cauterización con aguja monopolar de apertura fistulosa.

el vértice del seno piriforme y pasan a través de la membrana cricotiroidea por debajo del nervio laríngeo superior, siendo ésta una característica clave para lograr la distinción entre ambas ${ }^{12}$. Estas anomalías constituyen una alteración infrecuente, la última revisión sistemática de anomalías de cuarto arco branquial identificó 526 casos reportados, correspondiendo la mayoría de las publicaciones a series pequeñas de casos o reportes de caso único ${ }^{13}$.

La presentación clínica de las anomalías del cuarto arco branquial es variada. En pacientes pediátricos puede involucrar dificultad respiratoria 
y también se han descrito casos de fístulas cervicales cutáneas ${ }^{14,15}$. Sin embargo, en la mayoría de los casos se presenta mediante una masa cervical fluctuante, absceso cervical o fístula cutánea ubicada en el borde anterior del músculo $\mathrm{ECM}^{16}$. Otra forma frecuente de presentación clínica es mediante un cuadro de tiroiditis aguda supurativa. Taylor y cols $^{16}$ reportaron que $83 \%$ de los casos de tiroiditis aguda supurativa son izquierdos, $66 \%$ de ellos ocurrió tras una infección de vía respiratoria alta y $39 \%$ recurrió tras el tratamiento ${ }^{17}$; esto ha sido motivo de recomendación de estudio de anomalías de hendidura branquial en todos los casos de tiroiditis aguda supurativa ${ }^{18,19}$. Aproximadamente $83 \%$ a $97 \%$ de las anomalías de cuarto arco branquial son izquierdas ${ }^{4,16}$. Se ha sugerido que esto se debería al desarrollo vascular asimétrico ${ }^{20}$.

El diagnóstico tardío de anomalías del cuarto arco branquial demuestra su naturaleza benigna y también la dificultad de realizar el diagnóstico. Dentro de los estudios diagnósticos se encuentra el TAC de cuello con contraste, la resonancia magnética la cual es particularmente útil para detectar anomalías quísticas, la laringoscopía directa y el esofagograma con bario, el cual puede fallar durante la fase inflamatoria aguda ${ }^{8}$.

En contraste con este último, la laringoscopía directa puede ser realizada durante un episodio agudo con una serie de ventajas, entre las cuales se incluyen la identificación de una posible apertura fistulosa en el seno piriforme, la identificación de la fístula durante la exploración cervical al introducir sondas, catéteres, medios de contraste 0 mediante transiluminación del trayecto, y además la posibilidad de otorgar un tratamiento definitivo mediante cauterización. Por estas razones, se recomienda realizar una laringoscopía directa ante la sospecha de una anomalía de cuarto arco branquia| ${ }^{21}$. Se describe también que al realizar la maniobra de valsalva durante una tomografía computarizada o un esofagograma con bario, podría ayudar a abrir el trayecto sinusal tornándose éste más visible ${ }^{22,23}$. Además queremos destacar la utilidad del estudio con nasolaringofibroscopía en nuestra serie, mediante el cual fue posible observar edema del seno piriforme comprometido en dos de los casos, lo que orientó aún más la sospecha diagnóstica.
En la literatura se describen distintas alternativas terapéuticas. El manejo realizado con mayor frecuencia es el drenaje quirúrgico de la colección mediante cervicotomía. No obstante, esta alternativa presenta una alta tasa de recurrencia. Por otro lado, la resección quirúrgica de la lesión branquial, particularmente cuando se ha asociado a tiroidectomía parcial ha presentado tasas de recurrencia significativamente menores, sin embargo, este manejo presenta mayores tasas de complicaciones particularmente en menores de 8 años ${ }^{13}$. El tratamiento endoscópico, reportado en 62 casos $^{13}$, representa una técnica mínimamente invasiva en la cual se cauteriza y oblitera la apertura del trayecto fistuloso en el seno piriforme ${ }^{13,23}$. Esta técnica ha demostrado tasas de recurrencia similares respecto a cirugía abierta de cuello y resección de la lesión, con menores tasas de complicaciones y menor costo ${ }^{13}$.

Nuestros tres casos presentan características clínicas similares. Todos corresponden a pacientes masculinos jóvenes (3, 18 y 11 años), que presentaron un cuadro caracterizado por aumento de volumen cervical anterior izquierdo en contexto de una infección de vía respiratoria alta. Los 2 primeros casos fueron tratados mediante drenaje quirúrgico en primera instancia, ambos con recurrencia de absceso cervical entre los 7 y 10 meses posoperatorios. En todos los casos se pudo observar la apertura fistulosa en el vértice del seno piriforme izquierdo mediante la laringoscopía directa. Una vez realizada la cauterización de la apertura fistulosa con aguja monopolar, asociada a tratamiento antibiótico endovenoso, los pacientes evolucionaron de manera favorable sin recurrencia hasta la fecha con 32, 7 y 6 meses de seguimiento respectivamente.

\section{CONCLUSIONES}

Las anomalías de cuarto arco branquial constituyen una patología infrecuente. El diagnóstico se realiza mediante la historia clínica y estudios diagnósticos, entre los cuales la laringoscopía directa presenta una serie de ventajas.

El manejo endoscópico ha demostrado ser una alternativa segura y efectiva con una tasa de recurrencia comparable al manejo quirúrgico abierto, y menor tasa de complicaciones. 


\section{BIBLIOGRAFIA}

1. COHEN JR MM. Malformations of the craniofacial region: evolutionary, embryonic, genetic, and clinical perspectives. Am J Med Genet 2002; 115: 245-68.

2. Ascherson FM. De fistulis colli congenitis adjecta fissurarum branchialium in mammalibus avibusque historia succincta. Berolini: C.H. Jonas; 1832. p. 1-21.

3. Raven RW. Pouches of the pharynx and oesophagus with special reference to the embryological and morphological aspects. $\mathrm{Br} \mathrm{J}$ Surg 1933; 21: 235-56.

4. TuCKeR HM, Skolnick MI. Fourth branchial cleft (pharyngeal pouch) remnant. Trans Am Acad Ophthalmol Otolaryngol 1973; 77: 368-71.

5. Takal SI, Miyauchi A, Matsuzuka F, et al. Internal fistula as a route of infection in acute suppurative thyroiditis. Lancet 1979; 1: 751-2.

6. Pearce en, Farwell AP, Braverman Le. Thyroiditis. N Engl J Med 2003; 348: 2646-55.

7. Jeyakumar A, Hengerer AS. Various presentations of fourth branchial pouch anomalies. Ear Nose Throat J 2004; 83: 640-4.

8. Nicollas R, Guelfucci B, Roman S, et al. Congenital cysts and fistulas of the neck. Int $J$ Pediatr Otorhinolaryngol 2000; 55: 117-24.

9. MS Godin, DB Kearns, SM Pransky, AB Seid, DB WILSON. Fourth branchial pouch sinus: principles of diagnosis and management. Laryngoscope 100 (2 Pt 1) (1990) 174-8.

10. J.A. Jordan, J.E. Graves, S.C. Manning, J.E. McClay, M.J. BIAvatı. Endoscopic cauterization for treatment of fourth branchial cleft sinuses. Arch Otolaryngol Head Neck Surg 1998; 124 (9): 1021-4.

11. Franciosi JP, Sell LL, Conley SF, et al. Pyriform sinus malformations: a cadaveric representation. J Pediatr Surg 2002; 37: 533-8.

12. Keyvan N, Roland G, Harrison G, et al. Management of congenital fourth branchial arch anomalies: a review and analysis of published cases. Journal of Pediatric Surgery 2009; 44: 1432-9.
13. Yamakawa Y, Masaoka A, Kataoka M, et al. Mediastinal abscess caused by a pyriform sinus fistula: report of a case. Surg Today 1993; 23: 462-4.

14. Murdoch MJ, Culham JA, Stringer DA. Pediatric case of the day. Infected fourth branchial pouch sinus with an extensive complicating cervical and mediastinal abscess and left-sided empyema. Radiographics 1995; 15: 1027-30.

15. R.Y. Huang, E.J. Damrose, S. Alavi, D.R. Maceri, N.L. ShapIRo. Third branchial cleft anomaly presenting as a retropharyngeal abscess. Int J Pediatr Otorhinolaryngol 2000; 54 (2/3): 167-72.

16. W.E. Taylor JR., C.M. Myer III, L.L. Hays, R.T. CotTon. Acute suppurative thyroiditis in children. Laryngoscope 1982; 92 (11): 1269-73.

17. S.I. Takal, A. Miyauchi, F. Matsuzuka, K. Kuma, G. KosAKI. Internal fistula as a route of infection in acute suppurative thyroiditis. Lancet 1979; 1 (8119): 751-2.

18. M.E. Stone, D.T. Link, J.C. Egelhoff, C.M. MyeR III. A new role for computed tomography in the diagnosis and treatment of pyriform sinus fistula. Am J Otolaryngol 2000; 21 (5): 323-5.

19. Cho SS, ZalzAL GH. Branchial anomalies: a review of 52 cases. Laryngoscope 1995; 105: 909-13.

20. R.M. RosenfeLd, H.F. BILLER. Fourth branchial pouch sinus: diagnosis and treatment. Otolaryngol Head Neck Surg 1991; 105 (1): 44-50.

21. Mirauchi A, Tomoda C, Uruno T, et al. Computed tomography scan under a trumpet manoeuver to demonstrate piriform sinus fistulae in patients with acute suppurative thyroiditis. Thyroid 2005; 15: 1409-13.

22. TANAKA J. Usefulness of Vasalva's manoeuver during pharyngography in the diagnosis of acute suppurative thyroiditis: report of a case. Jpn J $f$ Clin Radiol 1996; 41: 559-61.

23. Verret DJ, McClay J, Murray A, et al. Endoscopic cauterization of fourth branchial cleft sinus tracts. Arch Otolaryngol Head Neck Surg 2004; 130: $465-8$.

Dirección: Mario Tapia C.

Servicio de Otorrinolaringología y Cirugía de Cabeza y Cuello, Hospital Guillermo Grant Benavente, Concepción, Chile.

E mail: mtapiac21@gmail.com 